Bangladesh Journal of Neuroscience 2018; Vol. 34 (1): 58-62

\title{
A Case of Adulthood Joubert Syndrome
}

\author{
HUQ MR ${ }^{1}$, SARKER I ${ }^{2}$, ISLAM MR ${ }^{3}$, HANNAN MA ${ }^{4}$
}

\begin{abstract}
:
Joubert Syndrome is a rare autosomal recessive disorder characterized by hypotonia, ataxia, breathing difficulties, developmental delay with hallmark molar tooth appearance in MRI. We report a rare case of adulthood Joubert Syndrome which is an unusual presentation. A 25 -years male presented to our outpatient department with developmental delay, dysphasia, ataxia, nystagmus, bilateral optic atrophy and hyperreflexia. MRI Brain showed classical 'molar tooth' appearance of cerebellar peduncles. Although an uncommon disorder, it is important to diagnose the condition early as physiotherapy and rehabilitation can be effective in coping with the symptoms causing developmental delay.
\end{abstract}

Key words: Joubert Syndrome, Hypotonia, Ataxia, Pigmentary Retinopathy, Developmental Delay etc.

\section{Introduction:}

Joubert syndrome (JS) was first described as a familial syndrome in 1969 by Marie Joubert in four siblings having agenesis of the cerebellar vermis presenting with episodic hyperpnoea, abnormal eye movements, ataxia and intellectual disability ${ }^{1}$. Several years later, midbrain-hindbrain malformation, the"molar tooth sign" (MTS), was detected first in JS and was considered as pathognomonic sign'2. The term "Joubert Syndrome and Related Disorders" (JSRD) was then introduced to describe all conditions sharing the $\mathrm{MTS}^{3}$, and this neuro-radiological sign is now considered as the mandatory criterion to diagnose JSRD. Many patients die in infancy or childhood due to marked breathing problems, but some survive into adulthood with variable cognitive and motor impairments ${ }^{4,5}$. Here, we discuss an adult case which was diagnosed principally by magnetic resonance imaging (MRI) finding of the typical "molar tooth sign", and by manifestation of other related clinical symptoms, including mental retardation, dysphasia, nystagmus and longstanding ataxia. To the best of our knowledge, this is the first report on an adult JS male individual in Bangladesh.

\section{Case Report:}

A 25 years old male attended our outpatient department with parental complaints on his difficulties in speech, walk and vision. According to his mother's statement, her child was floppy after birth, suggesting hypotonia. He also had history of frequent hospitalizations due to breathing difficulties in first few months of his life. As no documents are available, we can only assume that patient might have suffered from alternate apnea \& hyperpnoea. Patient had delayed milestones of development \& cannot speak at all. He also had progressive loss of his vision. Patient has five siblings, all are in good health and there is no significant family history including consanguinity. Patient did not receive any formal education but he could perform his own personal care activities, such as independent bathing, dressing and toileting. Physical examination showed deviation of head to right side, bilateral horizontal \& rotatory nystagmus with failure to abduct both eyes on

1 Dr. Muhammad Rezeul Huq, Resident, Department of Neurology, BSMMU, Dhaka, Bangladesh.

2 Dr. Imran Sarker, Registrar (Clinical Neurology), NINS\&H, Sher-E-Banglanagar, Dhaka, Bangladesh

3 Prof. (Dr.) Md. Rafiqul Islam, Chairman, Department of Neurology, BSMMU, Dhaka, Bangladesh.

4 Prof. (Dr.) M. A. Hannan, Professor, Department of Neurology, BSMMU, Dhaka, Bangladesh. 
lateral gaze (Figure 1,2). Patient doesn't interact properly and cannot speak at all. Visual acuity \& intelligence could not be assessed properly. Fundoscopy revealed bilateral optic atrophy with tessellated fundus (Figure3). Tone was normal in upper limbs, but increased in the form of spasticity in both lower limbs. Muscle power was normal. There was generalized hyperreflexia with bilateral ill-sustained ankle clonus. Plantar response was extensor bilaterally on Oppenheim's maneuver. Bilateral intention tremor was present with past pointing. Gait was broad based \& tandem gait was impaired. The patient, however, had no problems of breathing and other congenital anomalies. Axial MRI images obtained at the level of the midbrain showed a "molar tooth sign" typical in patients with JS (Figure 4). Further MRI image analysis revealed a bat wing-shaped fourth ventricle (Figure 5). Sagittal images additionally demonstrated hypoplasia of the vermis and dysgenesis of the corpus callosum (Figure 6). Putting all history, examination \& MRI findings together the patient was diagnosed with JS. Ultrasound examination of the abdomen revealed normal appearance of the liver, spleen, and both kidneys. His complete blood count, renal function tests, liver function tests, blood glucose, and serum electrolytes all were within normal limit. We were not able to conduct any genetic analysis for the patient and his family due to the circumstantial limits. The patient was later treated with physiotherapy \& other supportive management and his family was advised regarding general information on JS prognosis and patient support.

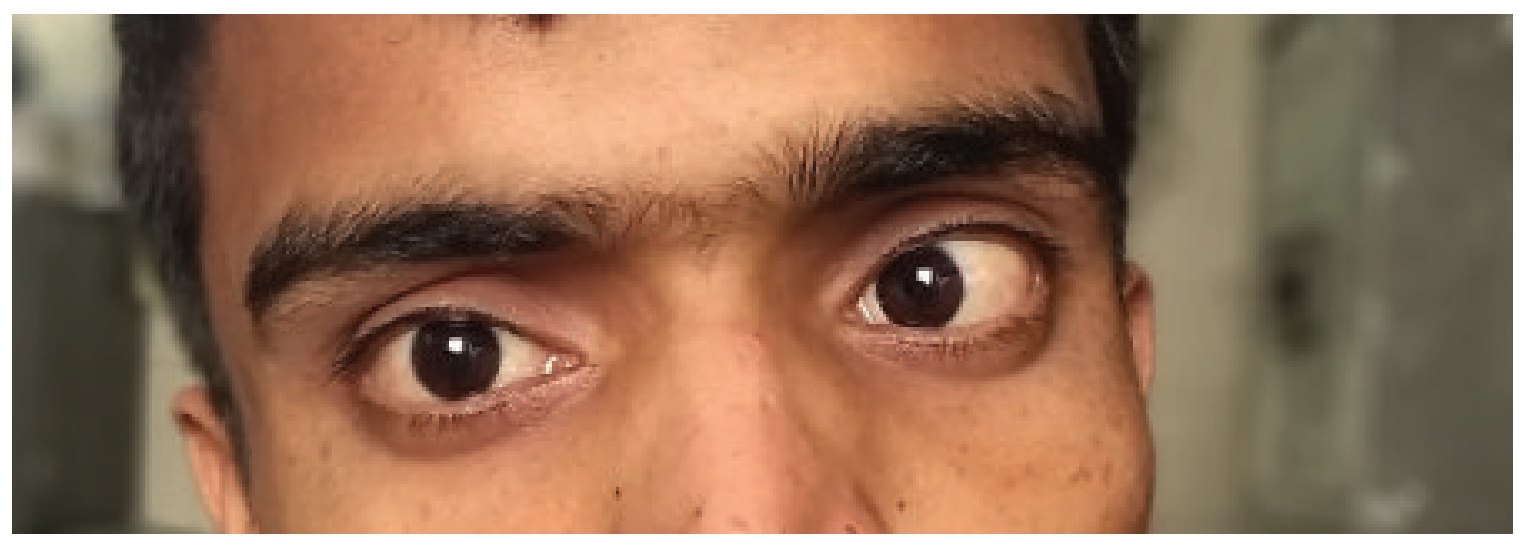

Fig.-1: On right lateral gaze, patient has abduction failure of right eye. Patient also has head tilting toward right side

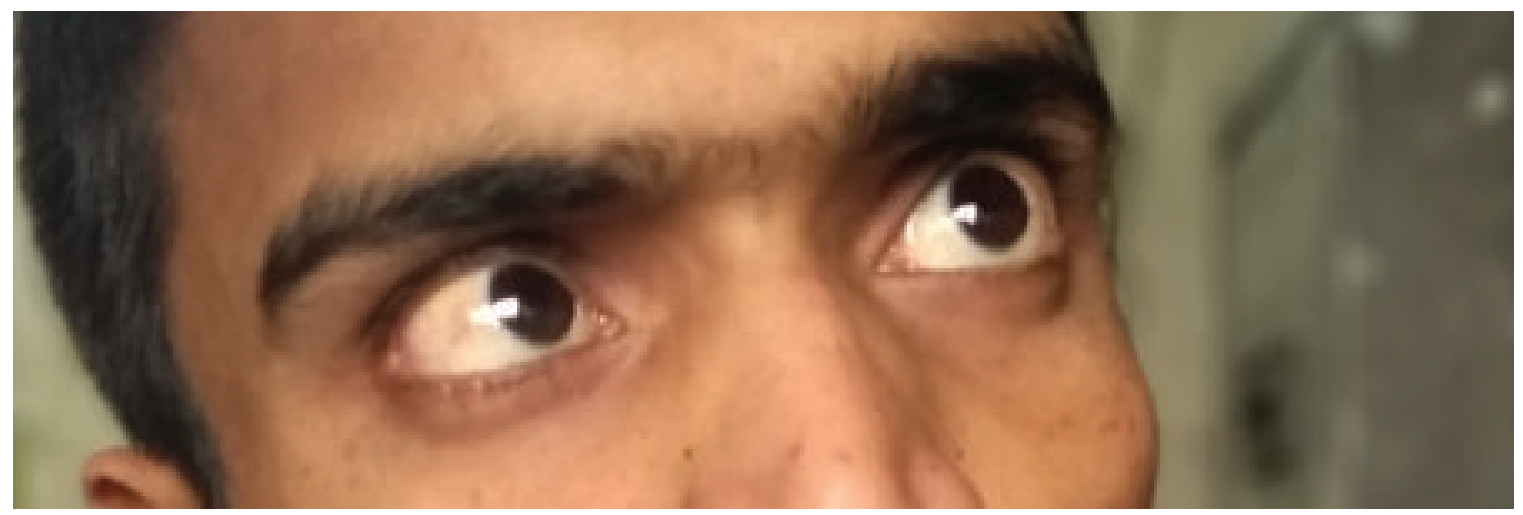

Fig.-2: On left lateral gaze, patient has abduction failure of left eye with persistent head tilting toward right side. 

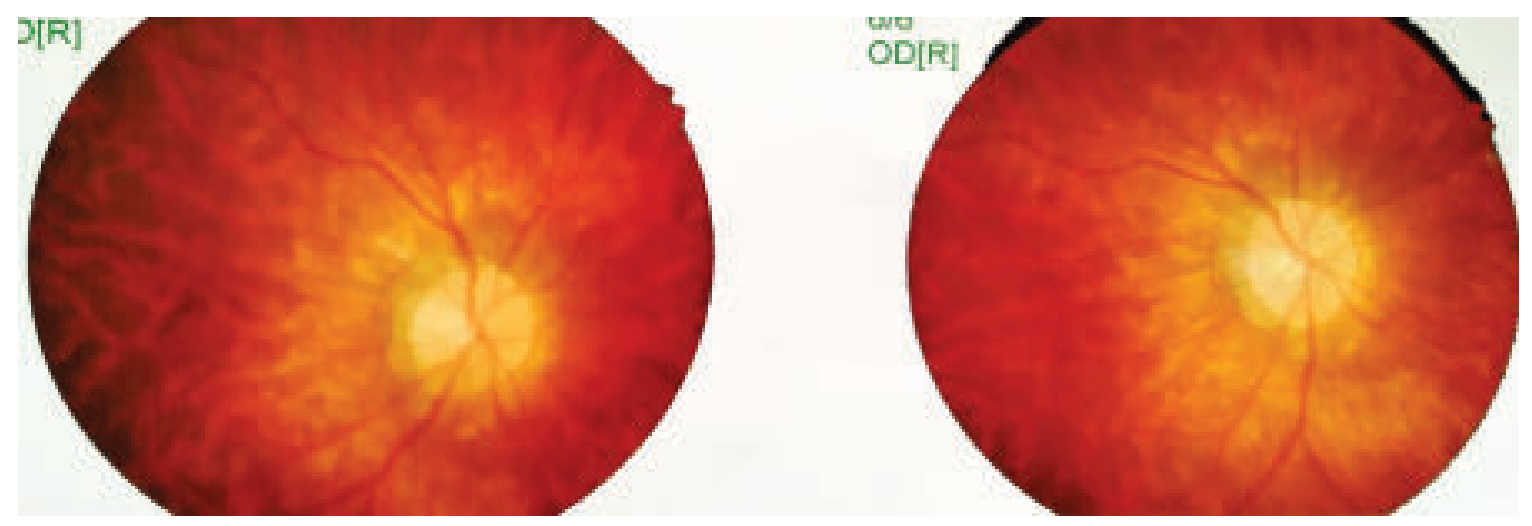

Fig.-3: Fundal photography showing bilateral optic atrophy with tessellated fundus

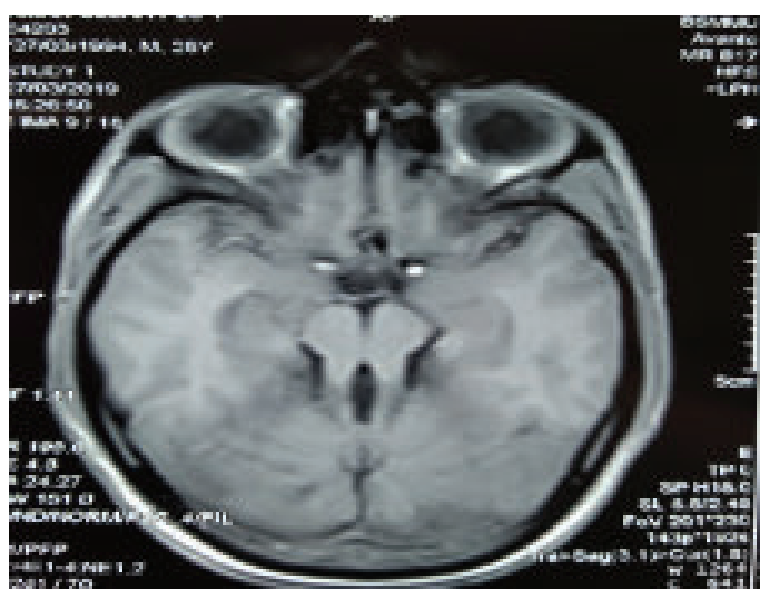

Fig.-4: Axial T1WIMRI section showing "molar tooth" appearance of the midbrain due to elongation and horizontal orientation of the superior cerebellar peduncles and the small midbrain. Note increased depth and decreased width of inter-peduncular distance.

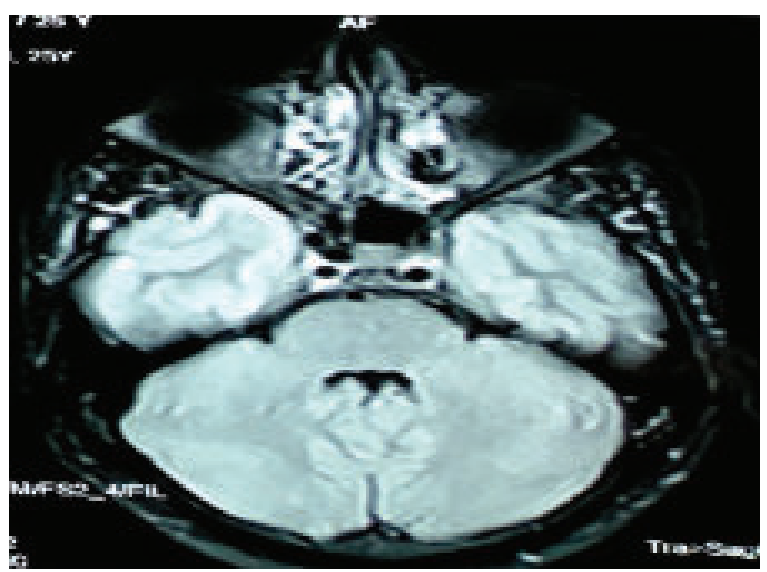

Fig.-5: Axial FLAIR MRI sequence showing bat wing appearance of fourth ventricle

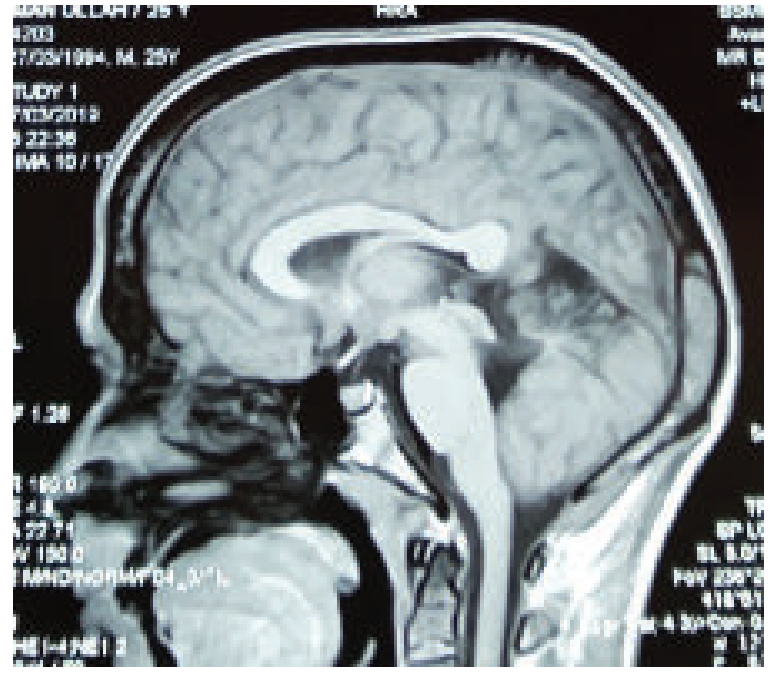

Fig.-6: Sagittal T1WI MR image shows marked hypoplasia of the cerebellar vermis with corpus callosum dysgenesis

\section{Discussion:}

JS is a rare autosomal recessive inherited disorder suspected by hypotonia, ataxia, developmental delay, intellectual disability with distinctive midhindbrain malformation (molar tooth sign), ocular abnormalities (e.g., pigmentary retinopathy, oculomotor apraxia, and nystagmus), renal cyst and hepatic fibrosis, hyperpnoea alternating with periods of apnoea ${ }^{6}$. Although the incidence of JSRD has not been precisely determined, it may range between $1 / 80,000$ and $1 / 100,000$ live births, but may be underestimated ${ }^{7}$. Though hypotonia at birth is a cardinal feature, in our patient we have found spasticity with generalized hyperreflexia, but 
patient was floppy at birth suggesting hypotonia. We have also found other two cases with hyperreflexia, though tone was not increased ${ }^{8,9}$. Ocular manifestations are also variable $\&$ there is a reported case with bilateral papilloedema also ${ }^{10}$.

The term "molar tooth" refers to the characteristic appearance of an enlarged and horizontally directed tubular structure on each side of the midline emerging from the midbrain. "The molar tooth sign" may be identified in $85 \%$ of patients with $\mathrm{JS}$, and thus can be considered pathognomonic of this disorder ${ }^{2}$.Inheritance of this disease is usually autosomal recessive, may be sporadic also. Studies have shown that it is a genetically heterogenous disorder with one locus pointing to chromosome $9 q^{11}$. In regions that lack genetic tests accessibility, neuroradiology should be used to assist final diagnosis ${ }^{12}$.

Once a diagnosis of JSRD has been made, children should undergo further evaluations to assess the possible multiorgan involvement. Ocular investigations include assessment of visual acuity, ocular motility, fundus and, whenever possible, electro-retinogram. Slit lamp examination can reveal abnormalities of the anterior segment of the eye. Kidney and liver functions should be tested. Standard urine analysis is also necessary including urine specific gravity. Abdominal ultrasound is necessary to evaluate the kidneys (to detect small cysts and loss of cortico-medullary differentiation), and the liver (to identify hepatomegaly or underlying congenital hepatic fibrosis) ${ }^{6}$.

Hence, there is no specific treatment for JS. Prognosis depends upon severity of symptoms and presence of associated anomalies but is generally poor due to the severe mental retardation in most cases. The main causes of death are severe feeding difficulties and respiratory infections with associated anomalies such as cranialmeningocele ${ }^{13}$. Respiratory abnormalities usually improve with increasing age of the patient, but cognitive and motor functions gradually decline ${ }^{13}$. Treatment for JS is therefore symptomatic and supportive. Multidisciplinary approach involving Infant stimulation, physical, occupational and speech therapy may have beneficial effects ${ }^{14}$.
Diagnosis in adulthood is still valuable as diagnosing underlying possible multiorgan involvement can prevent significant morbidity \& mortality.

\section{Conclusion:}

Today, it is still not possible to treat JS but it is possible to offer prenatal diagnosis and genetic counseling to those who have been diagnosed. Though commonly diagnosed in infancy \& childhood, some patients may be undiagnosed till adulthood. Diagnosis of these patients is also important as prognosis in adult JS depends mostly on renal and hepatic complications that, if not timely diagnosed and managed, represent the major causes of death in JSRD patients.

\section{List of abbreviations:}

JS: Joubert syndrome; MTS: molar tooth sign; JSRD: Joubert syndrome and related disorders.

\section{References:}

1. Joubert M, Eisenring JJ, Robb JP, Andermann F. Familial agenesisof the cerebellar vermis. Asyndrome of episodic hyperpnea, abnormal eyemovements, ataxia, and retardation. Neurology. 1969; 19:813-25.

2. Maria BL, Hoang KB, Tusa RJ, Mancuso AA, Hamed LM, Quisling RG, Hove MT, Fennell EB, Booth-Jones M, Ringdahl DM, Yachnis AT, Creel G, Frerking B. Joubert syndrome revisited: key ocular motor signs with magnetic resonance imaging correlation. Journal of Child Neurology.1997; 12:423-30.

3. Gleeson JG, Keeler LC, Parisi MA, Marsh SE, Chance PF, Glass IA, Graham JM, Maria BL, Barkovich AJ, Dobyns WB. Molar tooth sign of the midbrainhindbrainjunction: occurrence in multiple distinct syndromes. American Journal of Medical Genetics. 2004; 125A(2):125-34.

4. Steinlin M, Schmid M, Landau K, Boltshauser E. Follow-upin children with Joubert syndrome. Neuropediatrics. 1997;28(4): 204-11.

5. Gunzler SA, Stoessl AJ, Egan RA, Weleber RG, Wang P, Nutt JG . Joubertsyndrome surviving to adulthood associated with a 
progressivemovement disorder.Movement Disorders.2007;22:262-5.

6. Brancati F, Dallapiccola B, Valente EM. Joubert Syndrome andrelated disorders. Orphanet Journal of RareDiseases. 2010; 5:20.

7. Parisi MA, Doherty D, Chance PF, Glass IA.Joubert syndrome (and relateddisorders) (OMIM 213300)'European Journal of Human Genetics.2007; 15:511-21.

8. Zenger MN, KabatasS, BaykizAF, TengYD. A TurkishAdulthood Joubert Syndrome and Review of the Literature.European Journal of General Medicine. 2009;6(2):119-22.

9. Dahman $\mathrm{HAB}$, Mubaireek AHMB, Alhaddad $\mathrm{ZH}$. Joubert syndrome in a neonate: case report with literature review. Sudanese Journal Of Paediatrics.2016; 16(1):53-7.
10. Patel RJ, Patel V, Trivedi H, Parekh N.A rare case of Joubert syndrome. International Journal of Medical Science and Public Health. 2017;6(7):1237-9.

11. Chance PF, Cavalier L, Satran D, Pellegrino JE, Koenig M, Dobyns WB.Clinical nosologicand genetic aspects of Joubert and relatedSyndromes. Journal of Child Neurology. 1999;14: 660-66.

12. McGraw P. The Molar Tooth Sign.Radiology. 2003; 229: 671-2.

13. Suzuki T, Hakozaki M, Kubo N, Kuroda k, Ogawa A.A Case of cranial meningocele associated with Joubert Syndrome.Child's Nervous System. 1996;12(5): 280-2.

14. Spinella GM.Research directions: Follow up of the Joubert syndrome workshop. Journal of Child Neurology. 1999; 14: 667-72. 